\section{Der lange Weg zur Reform}

E. Taverna

Prof. Dr. med. Hannes Pauli hat von 1971 bis 1989 das Institut für Ausbildungsforschung (heute Institut für Aus-, Weiter- und Fortbildung IAWF) der med. Fakultät Bern geleitet und in dieser Zeit immer auch mit Gruppen von Studierenden am Krankenbett gearbeitet und gelegentlich Patienten mit psychosomatischen Problemen ambulant betreut. Das Institut war lange einzigartig in Europa und hat massgebend die Unterrichtsmethodik und -evaluation der Hochschulen, aber auch die Ziele der Allgemeinmedizin beeinflusst. Pauli wohnt heute im Altenberg über der Aare zusammen mit seiner Lebensgefährtin, der Regierungsrätin Dori Schaer. Die Ehefrau ist gestorben, die Kinder scheinen die vielen Interessen zu leben, die auch typisch für meinen Gesprächspartner sind: eine Tochter ist Psychiaterin, ein Sohn ist Theologe und Projektleiter in Afrika, der zweite Ethnologe, Journalist und Geschäftsleiter eines Restaurants mit einem kulturellen Veranstaltungsprogramm.

Hannes Pauli hat in Zürich studiert und zunächst als allgemeiner und chirurgisch-gynäkologischer Assistent in Rüti (ZH) und Glarus sowie als Praxisvertreter gearbeitet. Anschliessend hat er in den USA die Tuberkuloseabteilung einer Slumklinik in New York betreut, war in einem Belegspital in Pennsylvania als Resident tätig, um schliesslich im Henry-Ford-Spital in Detroit in der Kardiologie und Pneumologie klinisch und wissenschaftlich zu arbeiten. Er habilitierte 1964 in Innerer Medizin nach vorwiegend nephrologischer Tätigkeit an der Berner Medizinischen Poliklinik. Er hatte sich im Rahmen einer Arbeitsgruppe schon während fünf Jahren mit Ausbildungsreform-

Prof. Dr. med. Hannes Pauli

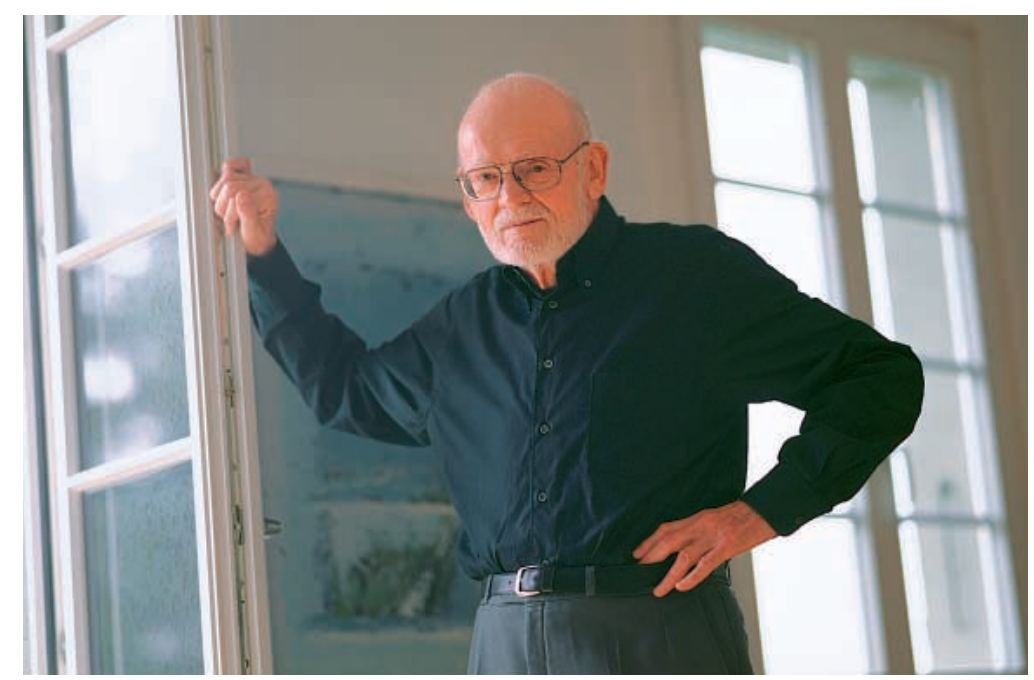

ideen befasst, als er von 1966 bis 1971 die Nachfolge Hadorns als Chefarzt und Mitglied eines Direktoriums von drei Personen übernahm. Als Experte für die medizinische Ausbildung der Fakultät lernte Pauli Gleichgesinnte in skandinavischen Ländern und in England kennen. Mit dem 1971, auf politischen Druck entstandenen und vom BAG finanzierten, von Pauli gegründeten Institut für Ausbildungsforschung wurde die internationale Zusammenarbeit, vor allem auch mit den USA, vertieft und auf einem Kongress «Medical Education and Primary Health Care» 1976 in Bern weltweit verbreitet. Nach der ersten Reform des Medizinstudiums in Kontinentaleuropa ab 1971 in Bern und anschliessend teilweise auch an den anderen schweizerischen Fakultäten sowie aus dem Vorhaben, das Staatsexamen in der Schweiz einheitlich durchzuführen (Neuformulierung der eidgenössischen Examensverordnung 1982), wurde dieses Reformmodell über viele Jahre in kleinen Schritten, mit langen Pausen weiter- und z.T. rückentwickelt; heute ist ein Neuanfang eingeleitet.

Vor und nach seiner Emeritierung 1989 pflegte H. Pauli internationale Kontakte zum Thema der Wissenschaftsentwicklung in der Medizin. Daraus ist 1990 die «Berne Group» mit Pionieren wie Kerr White (Public Health) und Mac Whinney (General Practice) hervorgegangen. Gemeinsam mit den letzteren hat Pauli im vergangenen Jahr in der Zeitschrift «Education for Health" Analysen und Thesen zu "Medical education, research, and scientific thinking in the $21^{\text {st }}$ century" programmatisch zusammengefasst.

Herr Professor, auf der Fahrt zu Ihnen war die Bahn voll von rüstigen, unternehmunglustigen AHVlern in roten Socken und Wanderschuhen. Das Land strotzt vor Gesundheit. Braucht da die Medizin einen Paradigmawechsel, wie Sie und Ihre Freunde behaupten?

Die erhöhte Lebenserwartung und Lebensqualität im Alter ist erfreulich. Unsere Umwelt ist aber nicht unbedingt gesund. Zum Beispiel die Arbeitswelt: darauf und andere gesellschaftliche Umstände weisen schon die Heerscharen von Psychologen und Psychiatern hin, die alle in diesem Bereich voll beschäftigt sind. Eine moderne Medizin muss Menschen helfen, sich ihrer heutigen Umgebung anzupassen. Dazu gehört auch eine Allgemeinmedizin, die unter allen Fachbereichen der Realität "Mensch und seine Umgebung" am nächsten steht; gesellschaftlicher Stress wirkt sich ja nicht nur auf der psychosozialen, sondern auch auf der somatischem Ebene aus. Die Fakultäten sollten deshalb Generalisten in der Ausbildung nicht nur zulassen, sondern an deren Leitung beteiligen (was in Bern teilweise der Fall ist).

Führen diese Ansprüche nicht zu einer enormen Ausweitung des Medizinbetriebs, von der schon Ivan Illich in den 70er Jahren gewarnt hat ("Die Enteignung der Gesundheit»)?

Ich habe Illich persönlich gekannt und mit ihm ausgiebig (u.a. auch kontrovers) diskutiert. Was ich suche und fordere, ist nicht eine Ausweitung der Medizin 
als Apparat, sondern eine wissenschaftliche Grundlage anhand der heutigen Erkenntnisse über die Natur und den Prozess der Gesundheit. Ich werde missverstanden, wenn dies mit "alternativer» Medizin gleichgesetzt wird. "Alternative» Ärzte haben vielfach eine erweiterte, ganzheitliche Sicht von ihren Patienten und deren Umgebung (wie wir sie befürworten), ihre Methoden und Techniken sind aber in vielen Fällen ebenso reduktionistisch und monokausal wie die naturwissenschaftlichen, die sie zu überwinden vorgeben. Auf der Seite der etablierten Medizin sind die technischen Entwicklungen gewiss faszinierend, ich habe schliesslich brillant operierte Augen und ein ebenso erfolgreich genähtes Mitralsegel. Problematisch aber sind die Verteilung der Mittel sowie die Konsumhaltung und damit verbunden die Kosten. Das britische und das niederländische Gesundheitswesen sind in dieser Beziehung überlegen, vor allem in der konsequenten Anwendung des Hausarztmodells. Die Misere im britischen Spitalsektor ist durch den staatlichen Geldmangel bewirkt; das gehört damit in ein anderes Kapitel. Die dort im ambulanten Bereich real existierende Medizin zeigt, dass unsere Vorstellungen von einem weniger fachorientierten medizinischen Curriculum durchaus umsetzbar wäre.

Sie fordern sehr radikal eine Neuverteilung der Macht, wobei Sie dem universitären Establishment und der Pharmaindustrie nicht sehr gewogen sind. Die Fakultäten sind in der heutigen multispezialistischen Zusammensetzung vielfach hilflos. Oft sind sie mit der politischen Führung in Auseinandersetzung. Für eine bessere Organisation gäbe es z. B. in den USA mehrere vorbildliche Modelle (allerdings auch andere, bedenkliche) mit einer klaren gesundheitspolitischen Ausrichtung dank einer diesbezüglich professionalisierten Leitung. Ich halte eine Modifikation der heutigen Hierarchie für notwendig. Die Familienmedizin müsste, zusammen mit den Vertreterinnen und Vertretern des "public health" und Angehörigen der nichtärztlichen Gesundheitsberufe, die Spezialisten dort beiziehen, wo sie aufgrund der Lernsituation benötigt werden. Sehen Sie, die Allgemeinmedizin ist ja heute an den Fakultäten nur am Rande vertreten. Ihre Vertreter sind viel zu bescheiden. Ich spreche hier nicht unbedingt von der "real existierenden" Allgemeinmedizin, sondern von einer Allgemeinmedizin, die in vieler Hinsicht noch Projekt geblieben ist. Dieser zentrale Arbeitsbereich ist ja vor der seit etwa 25 Jahren einsetzenden Erneuerung während vielen Jahrzehnten bei uns vernachlässigt worden.

An der Tagung der SAMW zu einer «Neu-Orientierung der Medizin" in Bern war sehr viel von einer neuen Moral und Ethik zu hören. Auch in Ihrer Arbeit über das neue Denken wird Weisheit und Liebe gefordert. Brauchen wir als neuen Arzt oder neue Ärztin einen periodisch zertifizierten Übermenschen? Nein, wir brauchen normale Menschen mit einem erweiterten Konzept von Leben, mit einem breiteren Bild der menschlichen Existenz. Unsere Studierenden haben heute keine Ahnung von den Vordenkern die- ser neuen Basis. Neue Ausbildungsziele hätten auch andere Inhalte zur Folge. Den oft additiv angehäuften und vorwiegend technischen Inhalten der etablierten akademischen Fächer sollte ein generalistisches Denken entgegengesetzt werden, wie es u.a. durch Jakob von Uexküll, Gregory Bateson, Ilya Prigogine, Thure von Uexküll, Humberto Maturana und Franzisco Varela angebahnt und konkretisiert worden ist. Ich plädiere für ein dem heutigen Stand der Entwicklung entsprechendes wissenschaftliches Erkenntnissystem. Gespräche mit erstjährigen Studenten zeigen eine hohe Bereitschaft, sich in dieses Neuland vorzuwagen. Die Realität des Studiums ist leider oft mit einer fortschreitenden Demotivation verbunden. Beispiele wie das Curriculum in Albuquerque, New Mexiko, zeigen den Weg. Dort liegt das Schwergewicht der Ausbildung v.a. im ersten Studienjahr bei den Praktikern der Region, die als Gegenleistung kostenlos mit dem Informationssystem der Fakultät und des Spitals elektronisch vernetzt sind. Meine Vision eines Curriculums beinhaltet ein derartiges Grundstudium, dessen Lehrinhalte alle biologischen, sozialen und psychologischen Erkenntnisse integrieren, die über das aktuell dominierende mechanistische Körperschema hinausweisen. Die Fleiner-Reform liegt darin sicher richtig, und ich beobachte auch in Bern durchaus derartige Ansätze. Die Gefahr sehe ich darin, dass die Inhalte weiterhin fächerzentriert vermittelt werden und sich damit die "frühkindliche Schädigung" der Studierenden aufgrund einer summativen, desintegrierten Anordnung weiter fortpflanzt.

Gibt es nicht bereits schon zahlreiche Belege für eine erweiterte Auffassung des biologischen Grundwissens, zum Beispiel auf dem Gebiet der Neurowissenschaften, wo ein Autor wie Gerhard Roth als Philosoph und Forscher hohes Ansehen geniesst?

Sie haben recht, Roth und andere Autoren, die ganze Systeme, vor allem das Neurosystem, beschreiben, gehen genau in die Richtung, die wir für die Lebenswissenschaften befürworten. Das Präfix «bio» ist dafür nicht mehr verwendbar, weil ihm paradoxerweise eine weitgehend auf die materiell-technischen Anteile begrenzte Bedeutung erteilt worden ist. Nur nutzen unsere Fakultäten dieses Potential leider viel zu wenig. Das Humansystem muss mit seinem Kontext immer der Ausgangspunkt bleiben. Als Epoche betrachtet wird damit das einseitig mathematischmaterialistische Paradigma abgelöst - oder besser: erweitert, ergänzt. Dieses Denken hat Jakob von Uexküll zu Beginn des vergangenen Jahrhunderts eingeführt, und dieses haben u.a. die bereits zitierten Autoren weiterentwickelt. Ich muss mich hier mit dem Hinweis auf sich selbst regulierende Systeme beschränken, in denen Informationen die wesentlichen Regulatoren biologischer Prozesse sind («Biosemiotik»). Das Konzept eines "Geistkörpers" als realistischer Rahmen für die Untersuchung von Lebensprozessen wird die Trennung von Natur- und Geisteswissenschaften zu überwinden helfen. Bei all diesen Perspektiven dürfen wir allerdings nicht ver- 
gessen, dass ein Grossteil der Kontakte und Handlungen in einer ärztlichen Praxis banal-alltäglich sind und keines weit angelegten Konzeptrahmens bedürfen, aber die hier diskutierten Ansätze sind die eigentliche Herausforderung.

Sie erwähnen in diesem Zusammenhang natürlich auch das Konzept der Salutogenese von Antonovsky (1923-1994) und beschreiben als Konsequenz ein Modell, "das Krankheit und Kranksein als Unfähigkeit einer Person oder eines Organismus interpretiert, Bedeutung aus der inneren und äusseren Umwelt zu generieren und deren Möglichkeiten und Angebote zu nutzen." Wollen Sie damit wie Dethlefsen und Dahlke in ihrem populären Buch "Krankheit als Weg" dem Kranken noch zusätzlich die Verantwortung für sein Leiden aufbürden?

Nein, das ist ein Missverständnis, das vielleicht auch auf unsere Wortwahl zurückzuführen ist. Mit «Unfähigkeit» meinen wir kein moralisches oder schuldhaftes Verhalten, sondern ein Nicht-imstande-Sein. Ärzte, Therapeutinnen müssen verstehen, welche Bedeutungen Patientinnen ihrem eigenen Dasein und ihrer Umgebung erteilen. Diese Bedeutungen können gesundheitsfördernd, "salutogen» oder schädlich, "pathogen" sein. Dies erstreckt sich über die materielle, mentale, energetische bis zur spirituellen Ebene. Das ärztliche Verstehen von Bedeutungserteilungen des Patienten ermöglicht Gespräche, Handlungen, Verschreibungen und Eingriffe, die selektiv eine Verstärkung der salutogenen Tendenzen versprechen. Dies ist dann einfach der medizinische Teil der Unterstützung und Begleitung in einer belastenden Lebenssituation. Praktisch geschieht natürlich vieles davon schon heute. Was fehlt sind Forschungsgrundlagen mit dem Ziel einer Bestätigung und Sicherung dieser Erkenntnisse. Es gibt wohl viele Einzelarbeiten, oft aus der Gegenposition von Aussenseitern heraus. Die von der öffentlichen Hand finanzierte Forschung ist aber verarmt. Die Industrie finanziert einen grossen Teil der Forschung als Ganzes und beeinflusst damit auch wesentlich die Forschungsinhalte. Was dabei herauskommt, illustriert u.a. anschaulich eine zunehmend biologische Ausrichtung der Psychiatrie, und ich fürchte, dass das politisch geforderte Sponsoring an den Universitäten diesen Trend noch verstärken wird. Ausdruck dieses Trends ist auch die rein naturwissenschaftlich-technischen Ausrichtungen der nationalen Forschungsschwerpunkte und die damit verbundene Vernachlässigung der Geisteswissenschaften.

Sie und Ihre Mitautoren fordern eine Umverteilung der Forschungsmittel und nennen ganz konkrete empirische Forschungsinhalte für Generalisten im ambulanten und hausärztlichen Versorgungsbereich, die das behauptete Kausalitätsnetz von Gesundheit und Krankheit analysieren.

Wir haben in unserer Arbeit exemplarisch auf 13 konkret mögliche Projekte hingewiesen, mit Themen wie Helicobacter pylori und Psychosomatik, Brustkrebs und Placeboeffekt bis hin zur Verbindung zwischen Spitalmilieu und Genesungsverlauf. Die deskriptive, klinische Forschung soll zum Verständnis von Phänomenen beitragen, die sich nicht in die konventionellen Krankheitskategorien der ICD einordnen lassen, die wesentlich die Verteilung der Gelder bestimmen. Die entsprechende Forschung wird den Bedarf an Ressourcen und Institutionen im Gesundheitswesen besser abschätzen lassen und damit eine erweiterte Medizin einleiten. Der Aufwand für die ärztliche Arbeit wird einerseits grösser sein, wenn es vermehrt darum geht, die Lebenssituation der betreuten Personen und vor allem deren Wahrnehmungen und ihre davon abgeleiteten Handlungsweisen zu verstehen. Andererseits wird eine tiefgreifende Reform der medizinischen Erziehung, der Forschung und des wissenschaftlichen Denkens nur dank diesem vermehrten Aufwand überhaupt möglich.

Herr Professor ich danke Ihnen für dieses Gespräch. 